\title{
COMPARISON OF WAVE AMPLITUDE, IMPLICIT TIME AND COMFORT LEVEL USING DAWSON-TRICK-LITZKOW, JET AND DENCOTT ELECTRODES IN ELECTRORETINOGRAPHY IN NORMAL ADULTS
}

\author{
Theresia Yinski PG ${ }^{1}$, M. Sidik ${ }^{1}$, Syntia Nusanti ${ }^{1}$, Aria Kekalih ${ }^{2}$ \\ ${ }^{1}$ Ophthalmology Department Medicine Faculty Universitas Indonesia \\ ${ }^{2}$ Community Medicine Department Medicine Faculty Universitas Indonesia
}

\begin{abstract}
Introduction: Several electrodes can be used in standard full-field electroretinography (ERG). However, there are no standard values for Indonesians yet. Therefore, this research aims to establish normal values, to compare them and to compare comfort levels using DTL, Dencott and Jet electrodes in Indonesian adults.

Methods: This was a correlative analytical study as well as a population descriptive study which took place in Ciptomangunkusumo Hospital over the course of April-August 2016. Through convenient sampling, 58 normal Indonesian subjects, age 19-49 years old were selected. ERG amplitudes and implicit time values were measured according to recommendations by the International Society for Clinical Electrophysiology of Vision (ISCEV). Evaluations consisted of scotopic 0.01, 3.0, OP and photopic 3.0 flicker. After examination, all subjects filled in a questionnaire about comfort levels, adopted from the visual analog scale of a 10 centimetre line. The score was later measured by ruler in centimetres.
\end{abstract}

Result: Pearson/Spearman correlation shows moderate to strong correlation between all parameters in the 3 electrodes $(r \geq 0.3)$. The paired t-test analysis showed a statistically significant difference in ERG normal values between electrodes with higher wave amplitudes and longer implicit times in Dencott and jet electrodes, compared to DTL electrodes $(p<0.05)$. Jet and DTL electrodes are more comfortable than Dencott electrodes for Indonesian adults in standard full-field electroretinography with scores of 5.21 (Dencott), 6.93 (Jet) and 7.64 (DTL).

Conclusion: DTL electrodes give the lowest wave amplitudes and the shortest implicit times and are the most comfortable electrode compared to Dencott and ERG jet electrodes, in standard full-field electroretinography in Indonesian adults.

Keywords: electroretinography, Dencott, DTL, Jet, electrode

Cite This Article: GONDOSARI, Theresia Yinski Pistari. Comparison of Wave Amplitude, Implicit Time and Comfort Level Using Dawson-Trick-Litzkow, Jet and Dencott Electrodes in Electroretinography in Normal Adults. International Journal of Retina, [S.I.], v. 3, n. 2, sep. 2020. ISSN 2614-8536. Available at: <https://www.ijretina.com/index.php/ijretina/article/view/123

\section{INTRODUCTION}

*Correspondence to:

Theresia Yinski PG,

Department of Ophthalmology

Universitas Indonesia,

cyinski@hotmail.com

Electroretinography is a very important type of examination since it can be used to evaluate the retina function objectively and can calculate the results in numbers. Electroretinography may serve as baseline data and follow up measurement for therapy, as well as a means to predict the prognosis of patients if surgery is considered.1,2 The International Society for Clinical Electrophysiology of Vision (ISCEV) recommends that every centre should have its own normal set of data because the characteristics of patient vary widely.3,4 Nowadays, there is quite a wide variety of electrode types 
which can be used for the electroretinography examination, such as scleral electrodes, corneal electrodes and eyelid electrodes. Good electrodes are important in order to produce a good electroretinography wave result. However, we should not forget to also consider the patient's comfortability in wearing the electrodes during the examination. Despite a lot of types of electrodes, the standard depends on the parameter used in the electroretinography examination and therefore there is no methods difference per electrodes usage. There are three main groups of electrodes which are scleral, corneal and eyelid electrodes.

In this research we limited the comparison to Dencott electrodes as the example of scleral electrodes, Jet electrodes as the example of corneal electrodes and Dawson-Trick- Litzkow (DTL) electrodes as the example of eyelid electrodes. This research aims not only to establish the normal values of standard full-field electroretinography at our hospital for the Indonesian population, but also to compare the values and comfort levels of using the 3 types of electrode in Indonesian adults.

\section{METHODS}

This study is a correlative analytical study which compares the normal results of standard full-field electroretinography as well as the comfort level of the electrodes usage in normal Indonesian adults. However, this study also serves as population descriptive study as it gives normal standard values for electroretinography examination in Indonesia. All 58 subjects, ages between 19-49 years old, were selected through convenient sampling and their normal status of both eyes were established through a series of examinations of visual acuity, intraocular pressure, Humphrey visual field, anterior segment evaluation, fundus photography, Ishihara colour blind test and Pelli-Robson contrast sensitivity test, over the course of April-August 2016. Subjects were excluded if they had high myopia ( $\geq 6$ dioptres), a history of intraocular surgery or laser treatment (photocoagulation), eye trauma, long-term drug usage or difficulty in pupil dilation.

The electroretinography examination was performed based on ISCEV recommendations. The order of electrode usage was randomised per subject, but all of the examinations was done on the same day for each subject by the same operator. ISCEV recommendations are as following:
1. Pupil dilation is done with tropicamide $1 \%$ prior to the examination

2. A drop of tetracaine $0.5 \%$ is used as topical anesthetics in both eyes

3. 20 minutes of dark adaptation is done prior to the placement of the electrodes (based on the randomisation order) and subjects are instructed to look straight forward to the fixation point in the machine dome for the scotopic 0.01 and 3.0 ERG and $3.0 \mathrm{OP}$

4. Afterwards, 10 minutes of light adaptation is done prior to the photopic fast flicker $30 \mathrm{~Hz}$ ERG

The questionnaire about comfort levels, adopting the visual analog scale of a 10 centimetre line, was given to the subjects after they had completed all examinations. The score was later measured by ruler in centimetres.

All procedures performed in this study involve human participants and therefore the procedures were in accordance with the ethical standards of the institutional research committee and with the 1964 Helsinki declaration and its later amendments or comparable ethical standards. This research has been approved by The Ethical Committee of the Medicine Faculty of Universitas Indonesia based on the Helsinki Declaration and ethic permission has been obtained. Informed consent was obtained from all individual participants included in the study.

\section{RESULTS}

The image below can further clarify the results of the standard full-field electroretinography examination.

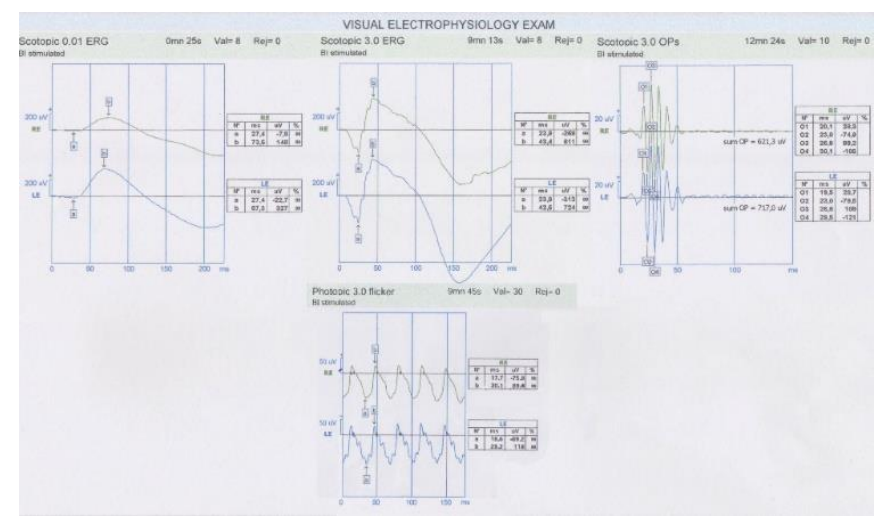

From the 58 subjects recruited, 43 were female, totaling 116 eyes. Pearson/Spearman correlation showed moderate to strong correlation between the three electrodes in all of the parameters measured, as given in table 1. 
Table 1. Correlation between Electrodes

\begin{tabular}{lccc}
\hline \multicolumn{1}{c}{ Parameter } & \multicolumn{3}{c}{ Correlation ( $\mathbf{r}$ ) } \\
\cline { 2 - 4 } & Dencott vs Jet & Dencott vs DTL & Jet vs DTL \\
\hline Implicit time of b wave Scotopic 0.01 & 0.626 & 0.609 & 0.650 \\
Amplitude of b wave Scotopic 0.01 & 0.604 & 0.436 & 0.572 \\
Implicit time of a wave Scotopic 3.0 & 0.737 & 0.476 & 0.487 \\
Amplitude of a wave Scotopic 3.0 & 0.564 & 0.358 & 0.388 \\
Implicit time of b wave Scotopic 3.0 & 0.615 & 0.582 & 0.641 \\
Amplitude of b wave Scotopic 3.0 & 0.826 & 0.668 & 0.649 \\
Implicit time of OP wave & 0.382 & 0.300 & 0.392 \\
Sum amplitude of OP wave & 0.787 & 0.584 & 0.688 \\
Implicit time of b wave Photopic Flicker & 0.623 & 0.565 & 0.636 \\
Amplitude of b wave Photopic Flicker & 0.640 & 0.467 & 0.584 \\
\hline
\end{tabular}

Normal values of standard full-field electroretinography in Indonesian adults, using Dencott electrodes, Jet electrodes and DTL electrodes, are shown in table 2. The $\mathrm{p}$-values were obtained from the paired t-test analysis.

Table 2. Normal Values of Standard Full-field Electroretinography in Indonesian Adults

\begin{tabular}{lccc}
\hline \multicolumn{1}{c}{ Parameter } & Dencott & Jet & DTL \\
\hline Imp.time b wave Scotopic 0.01 & $77 \pm 7$ & $76 \pm 6$ & $74 \pm 7^{* \wedge}$ \\
Amp. b wave Scotopic 0.01 & $293(133-544)$ & $332(109-576)^{*}$ & $283(146-496)^{\wedge}$ \\
Imp.time a wave Scotopic 3.0 & $25 \pm 1$ & $25 \pm 1$ & $24 \pm 1^{* \wedge}$ \\
Amp. a wave Scotopic 3.0 & $-258(-445 ;-138)$ & $-281(-459 ;-132)^{*}$ & $-220(-358 ;-100)^{* \wedge}$ \\
Imp.time b wave Scotopic 3.0 & $45 \pm 3$ & $46 \pm 4$ & $46 \pm 5$ \\
Amp. b wave Scotopic 3.0 & $482(315-737)$ & $509(261-771)^{*}$ & $417(202-724)^{* \wedge}$ \\
Imp.time OP wave & $21 \pm 1$ & $21 \pm 1$ & $21 \pm 1$ \\
Sum amp. OP wave & $345(106-589)$ & $338(149-797)$ & $281(107-717)^{* \wedge}$ \\
Imp.time b wave PhotoFlicker & $29 \pm 2$ & $29 \pm 2$ & $29 \pm 2$ \\
Amp. b wave PhotoFlicker & $105(44-236)$ & $108(44-264)$ & $88(37-186)^{*}$ \\
\hline
\end{tabular}

*) p value $<0.05$ compared with Dencott

^) p value $<0.05$ compared with Jet

The mean comfort level scores from the questionnaire using each electrode are shown in table 3.

Table 3. Mean Comfort Level Score of Electrode

\begin{tabular}{cc}
\hline Electrodes & Mean Comfort Level \\
\hline Dencott & $5.21 \pm 2.5$ \\
ERG Jet & $6.93 \pm 2.3$ \\
DTL & $7.64 \pm 1.8$ \\
\hline
\end{tabular}

The paired t-test comparison between the electrodes are shown in table 4.

Table 4. Comparison of Comfort Level between Electrodes

\begin{tabular}{cc}
\hline Electrode Comparison & p value \\
\hline Dencott - ERG Jet & 0.001 \\
Dencott - DTL & 0.001 \\
ERG Jet - DTL & 0.064 \\
\hline
\end{tabular}

There were no side effects or complications, such as eye infection or corneal abrasion, in any of the subjects during this research. 


\section{DISCUSSION}

Standard full-field electroretinography is an important clinical tool that provides an objective quantitative measure of retinal function. Decreased $a$ and $b$ wave amplitudes and prolonged implicit time correlate to reductions in retinal function. 1 Because of the wide variety of patient characteristics, ISCEV recommends that every laboratory should have its own normal data using ISCEV protocols so that it becomes comparable all over the world.1,3,4 However, until this time, there has been no research done in Indonesia regarding this matter.

Correlation analysis shows moderate to strong correlation between all parameters in the 3 electrodes. This explains that the higher the values are collected with Dencott electrodes, the higher the values will be collected with the two other electrodes. This result is similar to the research conducted in Japan by Kuze, et al. 5

The electrode order was randomised to eliminate the possibility of bias of eye fatigue or electrode type. The recording conditions also specify 20 minutes of dark adaptation before recording dark-adapted electroretinography (scotopic), and 10 minutes of light adaptation before recording light-adapted electroretinography (photopic) according to ISCEV standards. 6

This research shows that ERG jet electrodes give a higher amplitude than the other electrodes, with DTL giving the lowest. This happens because the DTL is quite far located from the centre of the cornea if compared with Dencott or Jet electrodes.5 However, the implicit times are not significantly different between electrodes, even though the material of each electrode is different. Silver, the material of DTL electrodes, has the highest conductivity of $6.8 \times 107(\Omega \mathrm{m})-1$, while gold, the material of Jet electrodes, has conductivity of $4.3 \times 107(\Omega \mathrm{m})-1.7$

In Malaysia and Iran adults the normal values of scotopic a and b wave amplitude is lower than in the Indonesian population when the recording is done by using DTL electrodes.8,9 Similar results are shown in research from Korea using Jet electrodes, which show lower results of wave amplitudes compared to the Indonesian population.10 However, it proved difficult to compare this research's results with other research, because the differences of electroretinography machines, as well as the electrodes, may contribute to a variety in results.

Race is known to be another factor that contributes to the difference of results. One research, comparing the Caucasian and Asian populations, showed that Caucasians tend to have a higher wave amplitude than Asians. The hypothesis of what cause this is said to be the ocular pigmentation of iris, in which melanine serves as a resistant barrier for the electroretinography wave signal. 11

The results from the questionnaires given to the subjects reveal that DTL electrodes give the most comfort of the three electrodes used. This is because of the position of the electrode, which is buried deep in the lower fornix, thus barely being in contact with the cornea. The electrode's position is stable as well, as the position is not necessarily affected by blinking of the eye. However, statistical analysis shows that there is no significant difference between DTL and Jet electrodes, which are shaped as contact lens touching the cornea.

The most ideal electrode is the one that give the highest amplitude, has low variability, and is welltolerated by the patients (most comfortable). The study by Mohidin N, et al shows that corneal electrodes such as Jet electrode may give a high variability because it tends to easily slide during blinking. 12 This makes the DTL as a non-corneal electrode a more favourable choice, as its variability is low due to its stable position.13 Dencott electrodes are the least favourable electrode due to their discomfort, caused by their bulky shape. However, its long legs make it stable, because once it is positioned well on the eye surface, it will not move even with rapid eye blinking.

In the future we hope to be able to conduct the examination with more varieties of electrodes on children as well as the geriatric subjects so that more complete normal standard values ERG can be obtained for all age population groups and more types of electrodes.

\section{CONCLUSION}

There is a statistically significant difference in the wave amplitudes between Dencott, Jet and DTL electrodes. DTL and ERG jet electrodes are the most comfortable electrodes in standard full-field electroretinography for normal Indonesian adults. Due to the presence of many 
parameters in the examination, the normal values of wave amplitude, implicit time and comfort levels of the three electrodes are given in the result section.

\section{REFERENCES}

1. Karanjia R, TenHove MW, Coupland SG. Electroretinograms and normative data. In: Belusic $\mathrm{G}$ (ed). Electroretinograms. Rijeka: InTech; 2011. p.1-238.

2. Miyake $Y$, Shinoda K. Clinical electrophysiology. In: Sadda SR, Ryan SJ (eds). Retina. $5^{\text {th }}$ ed. Philadelphia: Elsevier; 2013. p. 202-26.

3. Marmor MF, Zrenner E. Introduction to the iscev standards. In: Heckenlively JR, Arden GB (eds). Principles and practice of clinical electrophysiology of vision. $2^{\text {nd }}$ ed. London: The Mit Press; 2006. p. 287-8.

4. Marmor MF, Holder GE, Seeliger MW, Yamamoto S. Standard for clinical electroretinography (2004 update). Doc Ophthalmol. 2004; 108: 107-14.

5. Kuze M, Uji Y. Comparison between dawson, trick, and litzkow electrode and contact lens electrodes used in clinical electroretinography. Jpn J Ophthalmol. 2000; 44: 374-80.

6. McCulloch DL, Marmor MF, Brigell MG, Hamilton R, Holder GE, Tzekov R, et al. ISCEV standard for full-field clinical electroretinography (2015 update). Doc Ophthalmol. 2015; 130: 1-12.

7. Callister WD. Materials science and engineering: an introduction. $7^{\text {th }}$ ed. New York: John Wiley \& Sons, Inc; 2007. p. 674.
8. Parvaresh MM, Ghiasian L, Falavarjani KG, Sanjari MS, Sadighi N. Normal values of standard full field electroretinography in an iranian population. J Ophthalmic Vis Res. 2009; 4(2): 97-101.

9. Saiful AR, Chen AH, Muhamad SMR. Scotopic adapted full-field electroretinogram effect in young malay adults. IJERSTE. 2013; 2(6): 47-9.

10. Seong GM, Young $H L$, Sun YJ. Result of visualevokedpotential, electroretinography and electrooculography in normal subjects using monpack system. J Korean Ophthalmol Soc. 2014; 55(11): 1693 7.

11. Abdlseaed A, McTaggart $Y$, Ramage $T$, Hamilton R, McCulloch DL, Meigen T, et al. Light-and dark-adapted electroretinogram and ocular pigmentation: comparison of brown- and blue-eyed cohorts. Doc Ophthalmol. 2010; 121: 135-46.

12. Mohidin N, Yap MKH, Jacobs RJ. Electrodes for multifocal electroretinography: a comparison of four electrodes types. Sains Malays. 2014; 43(7): 1089-94.

13. Hébert $M$, Vaegan, Lachapelle P. Reproducibility of erg responses obtained with the $\mathrm{dtl}$ electrode. Vision Res. 1999; 39: 1069-70.

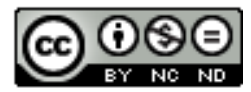

This work licensed under Creative Commons Attribution 